\title{
PULSATION MODES OF SPHERICAL STELLAR SYSTEMS
}

\author{
J. A. SELLWOOD \& C. PRYOR \\ Rutgers, the State University of New Jersey \\ Dept. of Physics and Astronomy, 136 Frelinghuysen Rd., Piscataway, NJ 08854-8019
}

\section{Introduction}

Kuhn \& Miller (1989, KM hereafter) proposed that a small stellar system in orbit around a large galaxy could be heated through resonant coupling between the orbital motion and internal modes of oscillation of the satellite. They further argued that the apparently large mass-to-light $(\mathrm{M} / \mathrm{L})$ ratios of dwarf spheroidal (dSph) galaxies around the Milky Way (e.g., Armandroff, Olszewski \& Pryor 1995) could be illusory because the satellites are far from virial equilibrium as a consequence of such resonant heating. We note that if the true M/Ls are small, some dSphs have much more kinetic energy than is required to unbind them; a resonant pumping mechanism which adds a modest amount of energy each cycle could not achieve this (Pryor 1996).

Nevertheless, KM raise two interesting issues. First, can stellar systems support large-amplitude pulsation modes? Second, if yes, can these internal modes be pumped by resonances with the orbit? We here confirm that certain low-concentration, spherical stellar systems support pulsation modes that are at most very weakly damped. We also report that we have been unable to excite these modes through orbital motion of the small stellar system in an external logarithmic potential.

Oscillations of spherical stellar systems have been studied using numerical simulations, beginning with the seminal paper by Hénon (1973) who was, however, most interested in instabilities. Systems which support weakly-damped oscillations were noted by David \& Theuns (1989), while Miller \& Smith (1994) report large-amplitude pulsations that appeared to be long-lived. Other models were constructed by Louis \& Gerhard (1988) and by Sridhar (1989). Mathur (1990) demonstrated the existence of discrete neutrâl pulsation modes in certain stellar systems and further work on 1-D systems is reported by Weinberg (1991) and by Louis (1992). It is clear from this work that oscillation modes can be sustained only when resonances between the mode and the frequencies of internal motion of the particles can be avoided.

\section{Damped and long-lived oscillations}

We have created a number of $N$-body realisations of different King-type and polytropic models of isolated spherical stellar systems. We searched for possible long-lived pulsations by disturbing the initial equilibrium in various ways and allowing the models to evolve. Although we tried a number of aspherical initial disturbances, we here focus on spherically-symmetric oscillations, since all of the long-lived oscillations that we have found so far are of this form.

Each of our 100000 particles moves in response to the accelerations determined from the global mass distribution. The gravitational field is determined through a surface harmonic expansion (to $l=4$ only) of the particle distribution in a large number (usually 200) of fixed spherical shells. All of the unperturbed models were in excellent equilibrium at the start and appeared to be stable.

We here present experimental results from a number of spherical models with isotropic velocity dispersions. These include a low-concentration King model and spherical stellar polytropes of different indices; such systems are described in detail in Binney \& Tremaine (1987, §4.4.3). The King model has $W_{0}=3$ and a tidal radius of $4.6994 \mathrm{King}$ radii. Quantities in this paper are given in units in which $G=M=a=1$; the unit of frequency is therefore $\left(G M / a^{3}\right)^{1 / 2}$. Here, $M$ is the total mass of the model and $a$ is either the King radius or the limiting radius for the polytrope. 

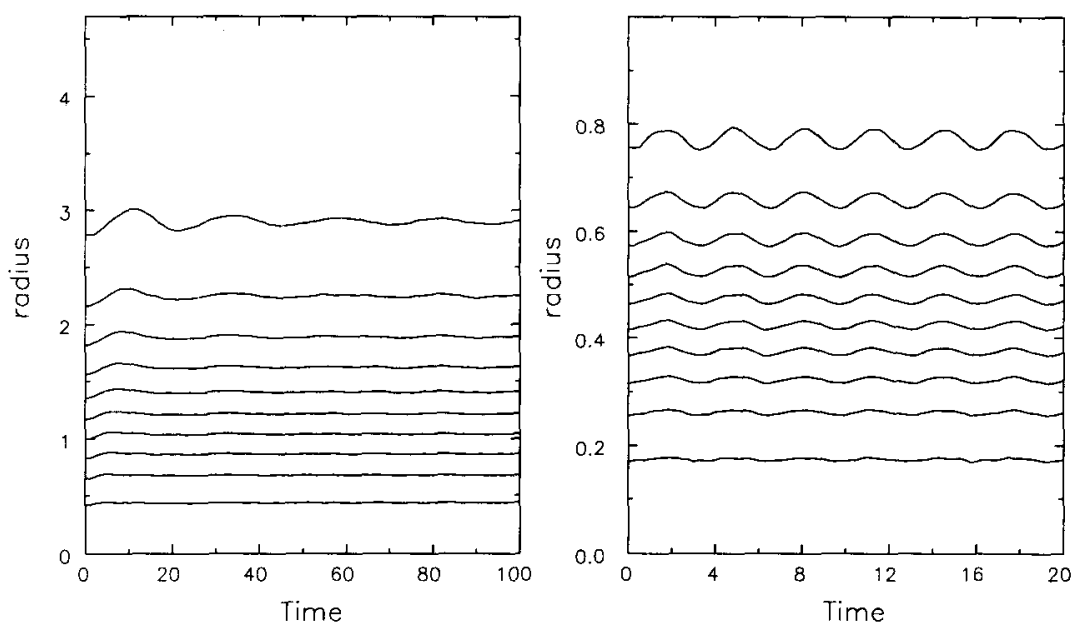

Figure 1. Radii containing various mass fractions of a $W_{0}=3 \mathrm{King}$ model (left) and an $n=2$ polytrope (right). The equilibrium of both models was disturbed at the outset.

Figure 1 shows the time evolution of radii containing $5 \%, 15 \%, \ldots, 95 \%$ of the mass of the King model on the left and of an $n=2$ polytrope on the right. In these cases, both models were disturbed by scaling the initial speeds of the particles so as to increase their kinetic energies near the center and to decrease them further out; the net result is a small increase in the overall kinetic energy. The disturbance to the King model decays quickly, while the polytrope appears to sustain a long-lived oscillation.

We have applied the mode-finding apparatus previously described by Sellwood \& Athanassoula (1986) to the time evolution of these models. We were able to obtain only a very rough estimate for the mode frequency in the King model, $0.35-0.21 i$, because the amplitude decayed so quickly. For the polytrope, on the other hand, we find a spherically symmetric pulsation mode of frequency 1.96 and no detectable decay rate.

We have found very weakly decaying modes in all polytropes with $n \leq 2.5$ and strongly damped modes in the more centrally concentrated polytropes with $n \geq 2.7$. Although the shapes of the eigenmodes resemble those predicted by Samimi \& Sobouti (1995), our frequencies do not agree closely with their predicted values.

The oscillation in the King model appears to decay through Landau damping by resonant particles. The nearly-horizontal line in each panel of Figure 2 marks the locus in energy, angular momentum $(E, L)$ space of the particles that have radial periods equal to our estimated period of the oscillation. Apparently, the very low-concentration polytrope can oscillate at a frequency low enough that no particle inside the truncation radius is in resonance, whereas there are resonant particles present in the King model, which presumably damp the oscillation.

Having determined the radial profile of the long-lived mode in the polytrope, we solved the radial continuity equation to determine a better initial perturbation that would excite the mode more cleanly. We tried a number of perturbations of increasing initial amplitude and found that the largest pulsation which we could excite in this manner caused the virial coefficient to oscillate by $\sim \pm 10 \%$ about 0.5 and the central density to vary by $\sim \pm 15 \%$. Pulsations from yet larger initial perturbations damped to about this amplitude.

\section{Orbit-mode coupling?}

We have performed a number of experiments in which we place our equilibrium $n=2$ polytrope into orbit in a fixed external logarithmic potential to search for possible coupling between the orbital motion and the internal oscillation mode. The orbital velocity was added to every particle and the grid recentered on the center of mass of the satellite at every step. 

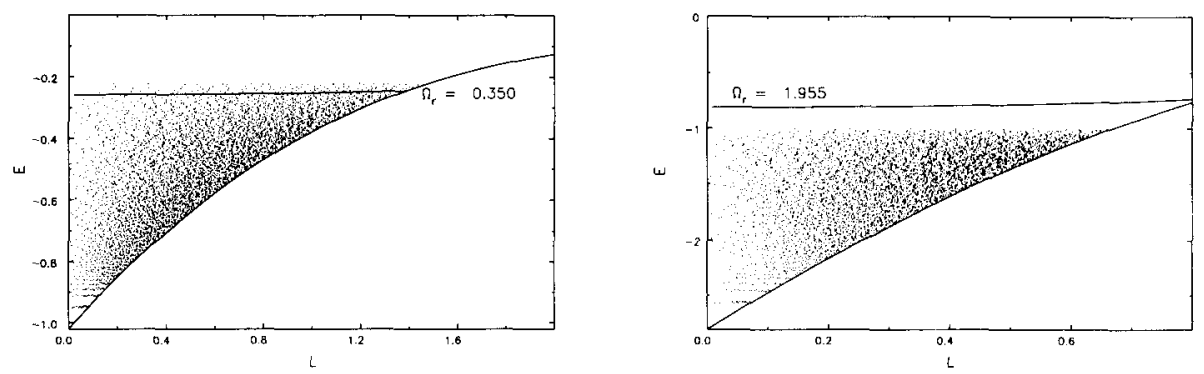

Figure 2. Undisturbed distributions of particles in ( $E, L)$-space for a $W_{0}=3 \mathrm{King}$ model (left) and an $n=2$ polytrope (right). The lower curved line marks the circular-orbit boundary. The dots indicate our initial particle distributions, which were selected with only 200 distinct $E$ values. The upper, more nearly horizontal line, marks the locus in this space of test particles having radial periods equal to the oscillation period we estimated from the models shown in Figure 1.

KM suggest that the mode can be excited if the satellite is on a circular orbit with a period equal to the pulsation period. However, these low-concentration models are easily disrupted by a tidal field and are therefore able to survive only if they orbit far from the center of a large galaxy. In an external logarithmic potential, we find that rapid tidal disruption occurs at any orbital radius inside where the orbital period is about twice the fundamental pulsation period of the polytrope. The 1:1 resonance envisioned by KM is not possible for these pulsations.

We tried a number of larger orbits, both circular and eccentric, but could not find one that led to significant excitation of the pulsation mode. We tried setting either the circular or epicycle frequency of the satellite's motion equal to a simple fraction of the pulsation mode frequency and saw no excitation. We also tried a circular frequency that would couple to the precession rate of the lines of apsides of orbits within the polytrope, again with no secular effects.

Since the pulsation mode to be excited is spherically symmetric, while the tidal field is bisymmetric, it is perhaps not too surprising that we were unable to excite it.

\section{Conclusions}

We have found that very low-concentration polytropes can support long-lived, spherical pulsation modes, while only slightly more centrally-condensed models do not. These systems can oscillate only if the pulsation frequency can avoid resonating with any particles; otherwise the mode is strongly Landau damped. We have so far found that $\sim 10 \%$ departures from virial equilibrium can be sustained, apparently indefinitely. We were unable to excite this mode through coupling to orbital motion of the satellite in an external potential and conclude that it is unlikely that the high M/Ls of the dSphs could be accounted for, even in part, by resonantly excited pulsation modes.

This work was supported in part by grants NSF AST 93/18617 and NASA Theory NAG 5-2803 (to JAS) and NSF AST 96/19510 (to CP).

\section{References}

Armandroff, T. E., Olszewski, E. W. \& Pryor, C. (1995) AJ, 110, 2131

Binney, J. \& Tremaine, S. (1987) Galactic Dynamics, Princeton University Press, Princeton

David, M. \& Theuns, T. (1989) MNRAS, 240, 957

Hénon, M. (1973) Astron. Ap., 24, 229

Kuhn, J. R. \& Miller, R. H. (1989) $A p J, 341$, L41

Louis, P. D. (1992) MNRAS, 258, 552

Louis, P. D. \& Gerhard, O. E. (1988) MNRAS, 233, 337

Mathur, S. D. (1990) MNRAS, 243, 529

Miller, H. H. \& Smith, B. E. (1994) Cel. Mech. Dyn. Astr., 59, 161

Pryor, C. (1996) in Formation of the Galactic Halo ... Inside and Out, H. Morrison \& A. Sarajedini, eds., ASP, San Francisco, p. 424

Samimi, J. \& Sobouti, Y. (1995) Astron. Ap., 297, 707

Sellwood, J. A. \& Athanassoula, E. (1986) MNRAS, 221, 195

Sridhar, S. (1989) MNRAS, 238, 1159

Weinberg, M. D. (1991) ApJ, 373, 391 